\section{Meningokokken: Nachholimpfung im Blick}

— Gemäß der Ständigen Impfkommission (STIKO) am Robert-Koch-Institut sollten Kleinkinder im zweiten Lebensjahr gegen Meningokokken des Serotyps C geimpft werden, z.B. mit dem Konjugatimpfstoff Menjugate ${ }^{\circledR}$ Kit. Da diese STIKO-Empfehlung erst im Jahr 2006 ausgesprochen wurde, ist die Trägerrate für C-Meningokokken bei Jugendlichen derzeit noch hoch. Deshalb werden Nachholimpfungen empfohlen und auch von den gesetzlichen Krankenkassen bezahlt.

In Großbritannien erfolgen bereits seit 1999 Impfungen gegen C-Meningokokken. Die Erfahrungen zeigen laut Prof. Dr. Ulrich Vogel, Würzburg, dass mit der Vakzinierung ein deutlicher Rückgang der Infektionen durch diesen Erreger erzielt werden konnte: Die Zahl nachgewiesener Infektionen nahm um den Faktor zehn ab. Ein impfbedingter Wechsel zu Erkrankungen mit anderen Meningokokkentypen („Switch“) wurde dabei nicht beobachtet.

Die Verbreitung einzelner MeningokokkenSerogruppen ist weltweit sehr unterschiedlich und variabel. „Das zeigt, wie wichtig die Entwicklung eines universalen Impfstoffs ist", betonte Vogel. Gegen die Serogruppen
A, C, W-135 und Y ist laut Vogel der neue tetravalente Konjugatimpfstoff von Novartis Behrung $\left(\right.$ Menveo $^{\circledR}$ ) wirksam - auch ohne Adjuvans. Der Antrag auf Zulassung wurde bereits bei der europäischen und US-amerikanischen Behörde (EMEA bzw. FDA) eingereicht. In der Zulassungsstudie (Perrett KP et al. 2009) erfolgte die Impfung zunächst

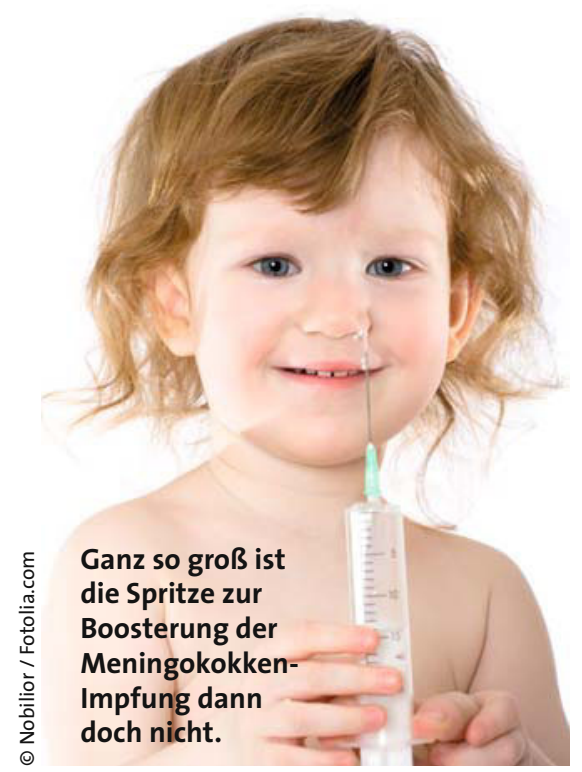

im zweiten und vierten Lebensmonat, dazu kam eine Boosterung im zwölften Monat. „Danach wurde ein sehr guter Schutz gegen alle Serogruppen erreicht", berichtete Vogel. Der Hersteller Novartis Behring plant noch weitere Impfinnovationen wie etwa einen rekombinanten Meningokokken-BImpfstoff, der sich in Phase III der klinischen Prüfung befindet. Meningokokken B sind weltweit die vorherrschende Serogruppe. PD Dr. Thomas Jelinek, Berlin, machte auf die Japanische Enzephalitis (JE) aufmerksam. In Asien versterben 20\% der infizierten Kinder und $50 \%$ der über 50-jährigen Patienten, Überlebende leiden häufig an permanenten Folgeschäden. In Japan ist die JE-Impfung seit 1967 Regelimpfung bei Kindern. Wie Jelinek berichtete, kam es aber aufgrund der Produktion unter Verwendung von Mausgewebe immer wieder zu systemischen Reaktionen. Eine neue Alternative ist Ixiaro ${ }^{\circledR}$, ein gut immunogen, aber wenig reaktogener Totimpfstoff, der zurzeit jedoch noch nicht für Kinder empfohlen wird.

Symposium , New Vaccines“ anlässlich der 101. Jahrestagung der Deutschen Gesellschaft für Tropenmedizin und Internationale Gesundheit. München, 6. November 2009. Veranstalter: Novartis Behring, Marburg

\title{
Option bei schwerer juveniler Psoriasis
}

_ Etwa 2-3\% der Bevölkerung in Deutschland leiden an Psoriasis. In 40\% der Fälle beginnt die Erkrankung bereits im Kindesund Jugendalter: Jeder Dritte erkrankt vor dem 16. Lebensjahr, jeder Zehnte vor dem 10. Lebensjahr. Wenn bei schweren Formen dieser systemisch-entzündlichen Erkrankung eine topische Therapie nicht mehr ausreicht, ist eine systemische Behandlung angezeigt. Immunsuppressiva wie Methotrexat, Ciclosporin oder Fumarsäureester sind bei Kindern und Jugendlichen jedoch nicht in randomisierten kontrollierten Studien erprobt.

Erst jetzt steht mit dem Biologikum Etanercept (Enbrel ${ }^{\circledR}$ ) ein systemisches Medikament zur Verfügung, dessen Effektivität in einer placebokontrollierten randomisierten doppelblinden Multizenterstudie bestätigt wurde. Der TNF-alpha-Blocker ist für die Therapie der chronischen schweren PlaquePsoriasis bei Kindern und Jugendlichen $a b$ dem achten Lebensjahr zugelassen, wenn andere systemische Therapien oder Phototherapie nicht ansprechen oder vertragen werden.

In die Zulassungsstudie (Paller AS et al. 2008) waren 211 Patienten im Alter von vier bis 17 Jahren mit mittelschwerer bis schwerer Plaque-Psoriasis eingeschlossen. Sie erhielten einmal wöchentlich Etanercept in der Dosis von 0,8 mg/kg Körpergewicht. Primärer Endpunkt der insgesamt 48-wöchigen Studie war die Verbesserung des Psoriasis Area and Severity Index (PASI) um mindestens $75 \%$ gegenüber dem Ausgangswert nach zwölf Wochen Behandlung.

Die Ergebnisse der Studie präsentierte deren Koautor, Prof. Dr. Richard Langley aus Halifax, Kanada. Nach zwölf Wochen zeigten $59 \%$ der Patienten die geforderte PASI-75-Response (11\% unter Placebo), nach 24 Wochen waren es $69 \%$. Die Hauterschei- nungen besserten sich bereits nach vier Wochen signifikant. Die Lebensqualität - gemessen als Veränderung im Children's Dermatology Life Quality Index (CDLOI) - zeigten nach zwölf Wochen gegenüber dem Ausgangswert einen Anstieg um 52\% (18\% unter Placebo).

Die Therapie mit dem Biologikum war sicher und verträglich. Unbekannte Nebenwirkungen traten auch in der Zulassungsstudie nicht auf. Die Infektionsrate war gering. Daten von Kindern mit juveniler idiopathischer Arthritis, für die Etanercept schon seit einigen Jahren zugelassen ist, zeigen auch für die Langzeittherapie keinen Anstieg schwerwiegender unerwünschter Ereignisse. koc

Pressekonferenz „Etanercept in der Behandlung der Plaque-Psoriasis bei Kindern und Jugendlichen" anlässlich der 45. Jahrestagung der Deutschen Dermatologischen Gesellschaft (DDG). Dresden, 1. Mai 2009. Veranstalter:Wyeth Pharma, Münster 